Supporting Information

\title{
Dye/Gelled Colloidal Photonic Crystal Composites and their Reversible pH-Responsive Optical Behaviors
}

\author{
Jian Dong and Weiping Qian*
}

State Key Laboratory of Bioelectronics, Department of Biological Science and Medical Engineering Southeast University, Nanjing 210096, People's Republic of China

* Corresponding Author: Fax: (+86)25-83795719, E-mail: wqian@ seu.edu.cn 
Figure S1 shows the different color of the two GCPCs. The GCPC is iridescent due to its structural color while the BB-doped GCPC is dark red due to its doped dye.

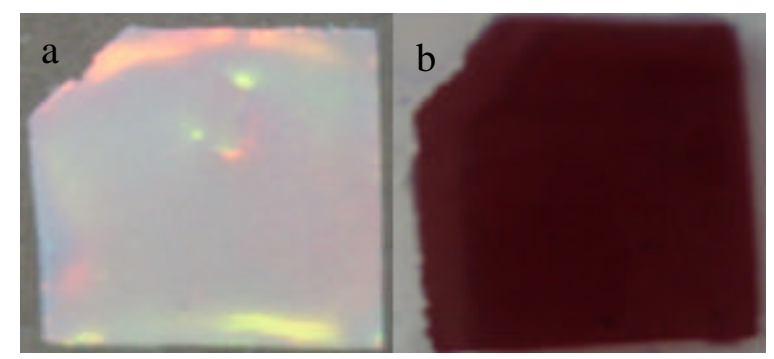

Figure S1. a) The image of a GCPC. b) The image of a BB-doped GCPC containing 20 g/l BB.

Figure $\mathrm{S} 2$ shows the absorption spectra of $\mathrm{BB}$ and Congo in both alkaline condition and acidic condition. In acidic condition, BB's extinction peak is at $435 \mathrm{~nm}$, and in alkaline condition, its extinction peak is at $592 \mathrm{~nm}$. In acidic condition, Congo red's extinction peak is at $497 \mathrm{~nm}$, and in alkaline condition, its extinction peak is at $572 \mathrm{~nm}$ and its width is more than $200 \mathrm{~nm}$.
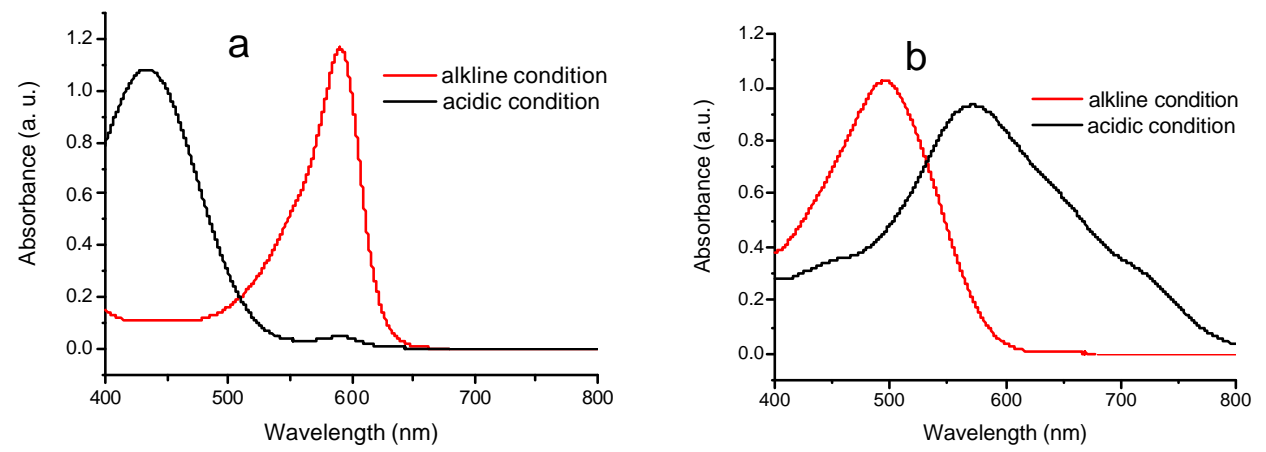

Figure S2. a) The absorption spectra of the BB. b) The absorption spectra of the Congo red. 
Suppose the light, ?, can be Bragg diffracted by the shorter Bragg diffracted layer of the GCPC. When it is incident through the shorter Bragg diffracted layer first, as shown in Figure S3a, the light is Bragg diffracted by the about 50 layers colloids of the shorter Bragg diffracted layer; When it is incident through the longer Bragg diffracted layer first, as shown in Figure S3b, the light Bragg is also diffracted by about 50 layers colloids of the shorter Bragg diffracted layer. Therefore in these two cases, the optical paths are different.

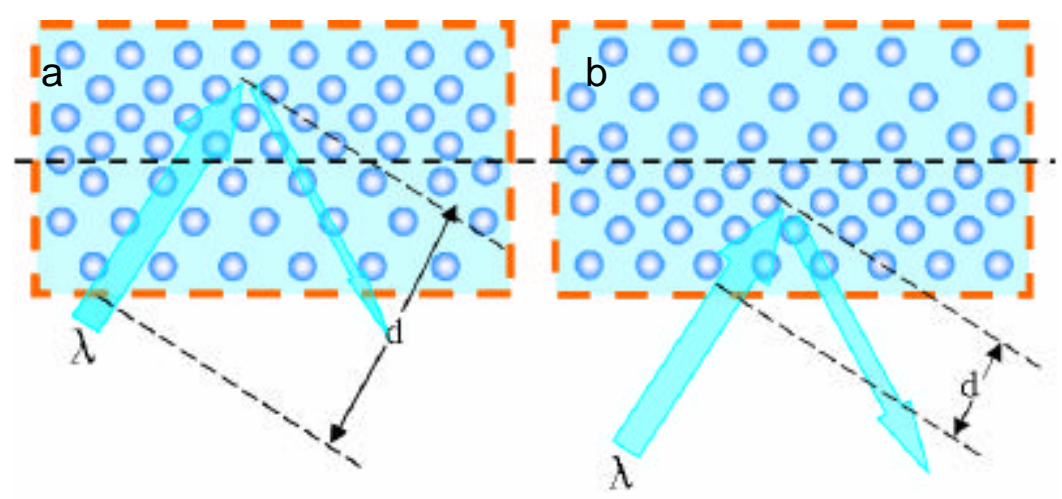

Figure S3. The illustration of the optical paths of light Bragg diffracted by the two layers of the GCPC, respectively. a): the top layer Bragg diffracted the incident light; b) the bottom layer Bragg diffracted the incident light. 
Figure S4 shows the images of the GCPC, in which the solutions $f$ acid and base are alternated and its color is also changed accordingly.

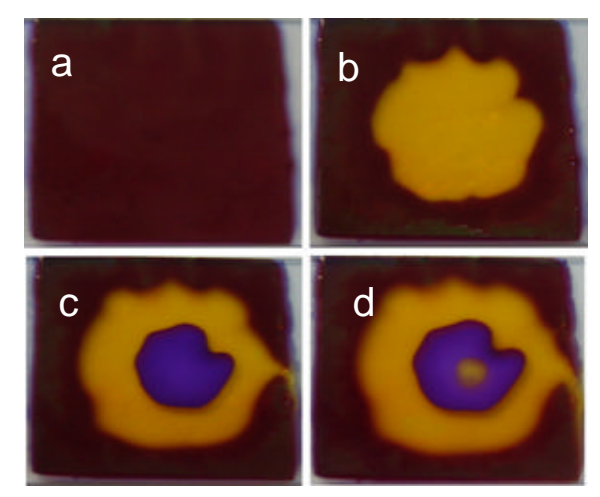

Figure S4. The images of the GCPC (a: Immersed and equilibrated in $25 \mathrm{~g} / 1 \mathrm{BB}$ aqueous solution; b: Adding $10 \mu \mathrm{l} 0.05 \mathrm{M} \mathrm{HCl}$ in the center of the GCPC in (a); c: Adding $5 \mu 1$ $0.05 \mathrm{M} \mathrm{NaOH}$ in the center of GCPC in (b); d: Adding $2.5 \mu 10.05 \mathrm{M} \mathrm{HCl}$ in the center of the GCPC in (c)). 